SHORT REPORT

\title{
Ultrastructural study of the extra-articular Leeds-Keio ligament prosthesis
}

\section{E Nomura, M Inoue, H Sugiura}

J Clin Pathol 2005;58:665-666. doi: 10.1136/icp.2004.024646

Background: There have been several histological studies of the Leeds-Keio ligament in anterior cruciate ligament reconstruction, but there have been few of the Leeds-Keio ligament in the extra-articular portion.

Aims/Methods: To report the histological and ultrastructural findings of two cases of medial patellofemoral ligament reconstruction using the Leeds-Keio ligament, removed 6.1 years and 8.7 years after implantation.

Results: In both cases, the tissue over the Leeds-Keio ligament was a ligament-like tissue. Electron microscopy showed that the diameter of the collagen fibrils in the tissue over the Leeds-Keio ligament was unimodal in the case investigated 6.1 years after implantation but bimodal in the case investigated after 8.7 years.

Conclusions: The tissue over the Leeds-Keio ligament may continue to grow with prolonged periods of mechanical stress.

T he Leeds-Keio (LK) ligament (Neoligaments, Leeds, UK), which is made of polyester as an open weave mesh-like structure, was developed as an alternative anterior cruciate ligament. ${ }^{1}$ Several histological studies of reconstructed anterior cruciate ligament LK ligaments have shown that the fibroelastic response to the LK ligament usually lacks axial orientation inside or immediately adjacent to the artificial ligament. ${ }^{2}{ }^{3}$ We histologically examined the extraarticular reconstructed medial patellofemoral ligament (MPFL) using the LK ligament and a medial retinaculum slip coverage ${ }^{4}$ in 15 specimens, one to nine years after transplantation, and showed that the tissue over the artificial ligament was mature only in specimens taken more than five years after surgery. In addition, we found that it was extremely important to investigate the reconstructed MPFL using the electron microscope.

This report presents the histological and ultrastructural findings of two Leeds-Keio MPFL grafts gathered 6.1 years and 8.7 years after implantation.

\section{CASE REPORT}

Two female patients aged 14 and 26 years underwent extraarticular MPFL reconstruction using the tape-type LK ligament and medial retinaculum slip coverage for recurrent patellar dislocation. ${ }^{5}$ The LK ligament was placed through the patellar tunnel and the two bundles were fixed to the anatomical MPFL femoral attachment using double stapling. Then the medial retinaculum slip (10 $\mathrm{mm}$ wide and $6-8 \mathrm{~cm}$ long with the patellar side intact) was covered over the LK ligament. Biopsies of the reconstructed MPFL were performed at the time of staple removal (with consent), 6.1 years and 8.7 years after surgery, respectively. The small biopsies$1.5 \mathrm{~mm}$ wide, $6 \mathrm{~mm}$ long, and $4 \mathrm{~mm}$ deep-were performed parallel to the longitudinal axis at the middle of the ligament

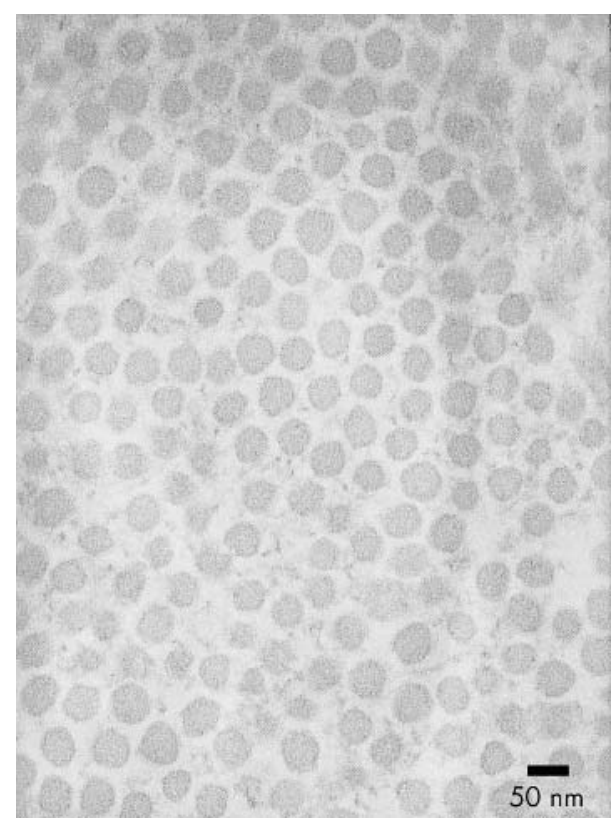

Figure 1 Case 1: 6.1 years after implantation (original magnification, $\times 100000)$. A unimodal distribution with small fibrils approximately $30-45 \mathrm{~nm}$ in diameter was seen.

and only the tissue over the LK ligament was selected for specimens. For light microscopy, the specimens were stained with haematoxylin and eosin and elastica von Gieson. For electron microscopic examination, a transmission electron microscope (JEM-1010, Nihon Densi, Tokyo, Japan) microscope was used.

\section{Pathology}

In both cases, sufficient connective tissue macroscopically ensheathed the whole prosthetic ligament, and the LK polyester fibres could not be seen from the surface. In both cases, light microscopy showed that the tissue over the LK ligament was made up of longitudinally aligned collagen fibre bundles with spindle shaped nuclei, hypovascularity, and crimp patterns. In the elastica von Gieson stained specimens, small numbers of elastic fibres were seen in the tissue over the LK ligament.

In both cases, transmission electron microscopy showed that the collagen fibrils in the tissue over the LK ligament were regularly orientated in the transversely sectioned area. However, collagen fibril diameter distribution was different in two cases. In case 1 (6.1 years after implantation), the collagen fibrils were uniform and approximately $30-45 \mathrm{~nm}$ in diameter (fig 1), whereas in case 2 (8.7 years after

Abbreviations: LK, Leeds-Keio; MPFL, medial patellofemoral ligament 


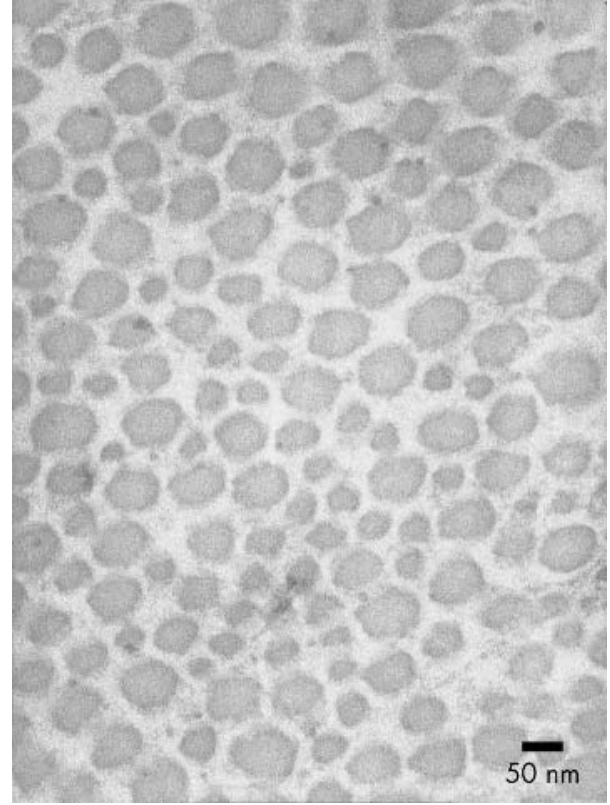

Figure 2 Case 2: 8.7 years after implantation (original magnification, $\times 100000$ ). Collagen fibrils had a bimodal distribution, with small fibrils approximately $25-40 \mathrm{~nm}$ in diameter and larger fibrils, approximately $50-70 \mathrm{~nm}$ in diameter.

implantation), the collagen fibrils were bimodal, and were approximately $25-40 \mathrm{~nm}$ in diameter and $50-70 \mathrm{~nm}$ in diameter (fig 2).

\section{DISCUSSION}

Many investigators have reported that reconstructed cruciate ligament grafts using autogenous tendons or allografts are histologically and biochemically similar to the normal anterior cruciate ligament one year after surgery. ${ }^{6-8}$ It is well known that the diameter of collagen fibrils in normal tendons or ligaments had a more or less pronounced bimodal distribution. ${ }^{9}$ However, many electron microscopic studies have shown that cruciate ligament autografts or allografts have a unimodal distribution, with small diameter collagen fibrils, and are different from normal tendons or ligaments, which have a bimodal distribution, even two years after surgery. ${ }^{810}$ Therefore, there has been no evidence of the reestablishment of a bimodal distribution of collagen fibrils in the reconstructed autografts or allografts.

\section{"Although the diameter of the larger collagen fibrils was not as large as that of large collagen fibrils in normal tendons and ligaments, this bimodal distribution of collagen fibrils is similar to that seen in the normal tendon and ligament"}

In our previous study, we showed that the tissue over the LK ligament seemed to be mature in specimens more than five years after implantation. In addition, we described the importance of the increased numbers of elastic fibres and that of the prolonged environment of mechanical stress in the development from a fibrotic tissue to a ligament-like tissue. In our present study, both patients, who had undergone surgery more than five years previously, also had matured connective tissue over the LK ligament on light microscopy. However, the two cases had different electron microscopic characteristics. In case 1 (6.1 years after implantation), collagen fibrils had a unimodal distribution, with small diameter fibrils of $30-45 \mathrm{~nm}$. In case 2 (8.7 years

\section{Take home messages}

- We investigated the histological and ultrastructural findings of two cases of medial patellofemoral ligament reconstruction using the Leeds-Keio (LK) ligament removed 6.1 years and 8.7 years after implantation

- In both cases, the tissue over the LK ligament was a ligament-like tissue, but electron microscopy showed that the diameter of collagen fibrils in the tissue over the LK ligament was unimodal 6.1 years after implantation and bimodal after 8.7 years

- The tissue over the LK ligament may continue to grow with prolonged periods of mechanical stress

after implantation), the collagen fibrils had a bimodal distribution, with smaller fibres of approximately $25-40 \mathrm{~nm}$ diameter and larger fibres, approximately $50-70 \mathrm{~nm}$ in diameter. Although the diameter of the larger collagen fibrils $(50-70 \mathrm{~nm})$ was not as large as that of large collagen fibrils in normal tendons and ligaments, this bimodal distribution of collagen fibrils is similar to that seen in the normal tendon and ligament. It is probable that the tissue over the LK ligament continues to grow with prolonged periods of mechanical stress.

\section{ACKNOWLEDGEMENTS}

Thanks to Mr Narutomi and Mrs Y Kato of the Department of Pathology of Kawasaki Municipal Hospital for assistance in preparing all the samples of light and electron microscopy.

\section{Authors' affiliations}

E Nomura, Department of Orthopaedic Surgery, Kawasaki Municipal Hospital, Kawasaki-ku, Kawasaki 210-0013, Japan

M Inoue, Department of Orthopaedic Surgery, Isehara-kyodo Hospital, Isehara, 259-1132, Japan

H Sugiura, Department of Pathology, Kawasaki Municipal Hospital

Correspondence to: Dr E Nomura, Department of Orthopaedic Surgery, Kawasaki Municipal Hospital, 12-1, Shinkawa-dori, Kawasaki-ku, Kawasaki 210-0013, Japan; edk-nomura@spn1.speednet.ne.jp

Accepted for publication 3 January 2005

\section{REFERENCES}

1 Fujikawa K, Iseki F, Seedhom BB. Arthroscopy after anterior cruciate reconstruction with the Leeds-Keio ligament. J Bone Joint Surg $\mathrm{Br}$ 1989;71:566-70.

2 Macnicol MF, Penny ID, Sheppard L. Early results of the Leeds-Keio anterior cruciate ligament replacement. J Bone Joint Surg Br 1991;73:377-80.

3 Prescott RJ, Ryan WG, Bisset DL. Histopathological features of failed prosthetic Leeds-Keio anterior cruciate ligaments. J Clin Pathol 1994;47:375-6.

4 Nomura E, Inoue M, Sugiura H. Histological evaluation of medial patellofemoral ligament reconstructed using the Leeds-Keio ligament prosthesis. Biomaterials 2005;26:2663-70.

5 Nomura E, Horiuchi Y, Kihara M. A mid-term follow-up of medial patellofemoral ligament reconstruction using an artificial ligament for recurrent patellar dislocation. Knee 2000;7:211-15.

6 Arnoczky SP, Warren RF, Ashlock MA. Replacement of the anterior cruciate ligament using a patellar tendon allograft. An experimental study. J Bone Joint Surg Am 1986;68:376-85.

7 Rougraff B, Shelbourne KD, Gerth PK, et al. Arthroscopic and histologic analysis of human patellar tendon autografts used for anterior cruciate ligament reconstruction. Am J Sports Med 1993;21:277-84.

8 Shino K, Oakes BW, Horibe S, et al. Collagen fibril populations in human anterior cruciate ligament allografts. Electron microscopic analysis. Am J Sports Med 1995;23:203-8.

9 Decker B, Bosch U, Kasperczyk W, et al. Ultrastructural changes of the patellar tendon as a cruciate ligament substitute (one year and two year results). J Submicrosc Cytol Pathol 1991;23:9-21.

10 Moeller HD, Bosch U, Decker B. Collagen fibril diameter distribution in patellar tendon autografts after posterior cruciate ligament reconstruction in sheep: changes over time. J Anat 1995;187:161-7. 